\title{
A Scalable, Accurate Hybrid Recommender System
}

\author{
Mustansar Ali Ghazanfar and Adam Prugel-Bennett \\ School of Electronics and Computer Science \\ University of Southampton \\ Highfield Campus, SO17 1BJ, United Kingdom \\ Email: \{mag208r,adb\}@ecs.soton.ac.uk
}

\begin{abstract}
Recommender systems apply machine learning techniques for filtering unseen information and can predict whether a user would like a given resource. There are three main types of recommender systems: collaborative filtering, content-based filtering, and demographic recommender systems. Collaborative filtering recommender systems recommend items by taking into account the taste (in terms of preferences of items) of users, under the assumption that users will be interested in items that users similar to them have rated highly. Content-based filtering recommender systems recommend items based on the textual information of an item, under the assumption that users will like similar items to the ones they liked before. Demographic recommender systems categorize users or items based on their personal attribute and make recommendation based on demographic categorizations. These systems suffer from scalability, data sparsity, and cold-start problems resulting in poor quality recommendations and reduced coverage. In this paper, we propose a unique cascading hybrid recommendation approach by combining the rating, feature, and demographic information about items. We empirically show that our approach outperforms the state of the art recommender system algorithms, and eliminates recorded problems with recommender systems.
\end{abstract}

Keywords-Recommender systems; collaborative filtering; content-based filtering; demographic recommender system.

\section{INTRODUCTION}

There has been an exponential increase in the volume of available digital information, electronic resources, and on-line services in recent years. This information overload has created a potential problem, which is how to filter and efficiently deliver relevant information to a user. This problem highlights a need for information extraction systems that can filter unseen information and can predict whether a user would like a given resource. Such systems are called recommender systems.

Let $M=\left\{m_{1}, m_{2}, \cdots, m_{x}\right\}$ be the set of all users, $N=\left\{n_{1}, n_{2}, \cdots, n_{y}\right\}$ be the set of all possible items that can be recommended, and $r_{m_{i}, n_{j}}$ be the rating of user $m_{i}$ on item $n_{j}$. Let $u$ be a utility function that measures the utility of item $n_{j}$ to user $m_{i}$, i.e.

$$
u: M \times N \rightarrow R,
$$

where $R$ is a totally ordered set. Now for each user $m_{i} \in$ $M$, the aim of a recommender system is to choose that item $n_{j}^{\prime} \in N$ which maximizes the user's utility [1]. We can specify this as follows:

$$
n_{j_{m_{i}}}^{\prime}=\underset{n_{j} \in N}{\arg \max _{i}} u\left(m_{i}, n_{j}\right): \forall_{m_{i} \in M}
$$

where the utility of an item is application dependent.

Collaborative Filtering (CF) systems can be classified into two sub-categories: memory-based CF and modelbased CF [1]. Memory-based approaches make a prediction by taking into account the entire collection of previous rated items by a user; examples include userbased CF algorithms [2]. Model-based approaches learn a model from collection of ratings and use this model for making prediction; examples include item-based CF [3] and Singular Value Decomposition (SVD) based models [4]. Model-based approaches are more scalable than userbased approaches.

There are two potential problems with the recommender systems. One is the scalability, which is how quickly a recommender system can generate recommendation, and the second is to ameliorate the quality of the recommendation for a customer. Pure CF recommender systems produces high quality recommendation than those of pure content-based and demographic recommender systems, however, due to the sparsity ${ }^{1}$, they can not find similar items or users using rating correlation, resulting in poor quality predictions and reduced coverage $^{2}$. Furthermore, All individual systems fail in cold-start [1] problems.

In this paper, we propose a hybrid scheme (namely Boosted $_{R D F}$ ) which produces accurate and practical recommendation and can be used in cold-start scenarios. Our proposed scheme is based on a cascading hybrid recommendation technique ${ }^{3}$ [5] that build item models based on item's rating, feature, and demographic information; and generates more accurate prediction than available state of the art recommender algorithms. We evaluate our algorithm on MovieLens ${ }^{4}$ and FilmTrust ${ }^{5}$ datasets.

\section{BACKGROUND}

\section{A. Item-Based Collaborative Filtering Recommender Sys- tems: Item Rating Information}

Item-based $\mathrm{CF}$ recommender systems [3] build a model of item similarities using an off-line stage. There are three main steps in these systems as follows:

\footnotetext{
${ }^{1}$ The percentage of ratings assigned by users is very small as compared to the percentage of ratings the system has to predict.

${ }^{2}$ The percentage of the items that can be recommended from all available items in the system.

${ }^{3}$ In cascading hybrid recommender systems, a recommendation technique is applied to produce a coarse candidate list of items for recommendation that are refined by applying other recommendation techniques.

${ }^{4}$ www.grouplens.org/node/73

${ }^{5}$ http://trust.mindswap.org/FilmTrust.
} 
Table I

A SAMPle OF FunCtions (F) With $k=20$

\begin{tabular}{|l|l|l|l|}
\hline Function \# & Function (f) & $\begin{array}{l}\text { MAE } \\
\text { (ML) }\end{array}$ & $\begin{array}{l}\text { MAE) } \\
\text { (FT) }\end{array}$ \\
\hline 1 & $R_{\text {ISim }}$ (Item-based CF) & 0.791 & 1.442 \\
\hline 2 & $F_{\text {ISim }}$ & 0.787 & 1.436 \\
\hline$\vdots$ & $\vdots$ & $\vdots$ & $\vdots$ \\
\hline 32 & $\vdots$ & $\mathbf{0 . 7 3 6}$ & $\mathbf{1 . 3 7 9}$ \\
$\vdots$ & $\vdots$ & $\vdots$ & $\vdots$ \\
\hline 83 & $\vdots$ & & \\
& $R_{\text {ISim }}+R_{\text {DSim }}+F_{\text {ISim }}+D_{\text {DSim }}+D_{\text {ISim }}$ & & \\
& $R_{\text {DSim }}+F_{\text {DSim }}+D_{\text {DSim }}$ & 0.834 & 1.451 \\
\hline
\end{tabular}

1- All items rated by an active user $^{6}$ are retrieved.

2- Target item $^{7}$ 's similarity is computed with the set of retrieved items. A set of $k$ most similar items, also known as neighbours of the target item with their similarities are selected.

3- Prediction for the target item is made by computing the weighted sum of the active user's rating on the $k$ most similar items.

\section{B. Content-Based Recommender Systems: Item Feature Information}

Content-based recommender systems [1] recommend items based on the textual information of an item. In these systems, an item of interest is defined by its associated features, for instance, NewsWeeder [8], a newsgroup filtering system uses the words of text as features.

We downloaded information about movies from IMDB $^{8}$, and applied $T F-I D F$ [8] approach for extracting the features from the information about each movie. After stop word removal and stemming, we constructed a vector of keywords, tags, directors, actors/actresses, and user reviews given to a movie in IMDB. Furthermore, we leverage WordNet using Java Wordnet Interface ${ }^{9}$ for overcoming the synonym problems between features while finding the similarities among (text) features.

\section{Demographic Recommender Systems: Item Demo- graphic Information}

Demographic recommender systems [6], [5] categorize users or items based on their personal attributes and make recommendation based on demographic categorizations. In our work, we used genre information about a movie as its demographic information and constructed a vector as used in [7].

\section{EXPERIMENTAL EVALUATION}

\section{A. Dataset}

We used MovieLens (ML) and FilmTrust (FT) datasets for evaluating our algorithm. MovieLens dataset contains 943 users, 1682 movies, and 100000 ratings on an integer scale of 1 (bad) to 5 (excellent). MovieLens dataset has

\footnotetext{
${ }^{6}$ The user for whom recommendation are generated.

${ }^{7}$ The item a system wants to recommend.

${ }^{8}$ www.imdb.com.

${ }^{9}$ http://projects.csail.mit.edu/jwi.
}

been used in many research projects [3], [4], [7]. The sparsity of this dataset is $93.7 \%\left(1-\frac{\text { non zero entries }}{\text { all possible entries }}=1-\right.$ $\left.\frac{100000}{943 \times 1682}=0.937\right)$.

we created the second dataset by crawling the FilmTrust website. The dataset retrieved (on 10th of March 2009) contains 1592 users, 1930 movies, and 28645 ratings on a floating point scale of 1 (bad) to 10 (excellent). The sparsity of this dataset is $99.06 \%{ }^{10}$.

\section{B. Metrics}

Our specific task in this paper is to predict scores for items that already have been rated by actual users, and to check how well this prediction helps users in selecting high quality items. Keeping this into account, we use Mean Absolute Error (MAE) and ROC (Receiver Operating Characteristic) sensitivity.

$M A E$ measures the average absolute deviation between a recommender system's predicted rating and a true rating assigned by the user. It is computed as follows:

$$
M A E=\frac{\sum_{i=1}^{N}\left|r_{p_{i}}-r_{a_{i}}\right|}{N},
$$

where $r_{p_{i}}$ and $r_{a_{i}}$ are the predicted and actual values of a rating respectively, and $N$ is the total number of items that have been rated. It has been used in [2], [3].

ROC sensitivity measures the probability with which a system accept a good item. The ROC sensitivity ranges from 1 (perfect) to 0 (imperfect). For MovieLens dataset, we consider an item good if the user rated it with a score of 4 or higher and bad otherwise. Similarly, for FilmTrust dataset, we consider an item good if the user rated it with a score of 7 or higher and bad otherwise. It has been used in [9].

Furthermore, we used coverage that measures how many items a recommender system can make recommendation for. It has been used in [10].

\section{Proposed Algorithm}

Let $m_{a}, n_{t}, R, D, F$ be the active user, target item, useritem rating matrix [1], demographic vector for an item $n_{j}$, and feature vector for an item $n_{j}$ respectively. Let $R_{I \text { Sim }}$, $D_{\text {ISim }}$, and $F_{I \text { Sim }}$ represent the rating, demographic, and feature similarity among the items. Furthermore, let $R_{D \text { Sim }}, D_{D \text { Sim }}$, and $F_{D \text { Sim }}$ represent the rating similarity among candidate items found after applying the feature correlation among all items, demographic similarity among candidate items found after applying the feature correlation among all items, and feature similarity among candidate items found after applying the rating correlation among all items.

The proposed algorithm can be summarized as follows:

Step-1: Compute the similarity between items using rating data, demographic data, and feature data, and store

\footnotetext{
${ }^{10}$ Both datasets can be downloaded from https://sourceforge.net/projects/hybridrecommend.
} 
Table II

A SAMPLE OF PARAMETER SET WITH $k=20$

\begin{tabular}{|l|l|l|l|l|l|}
\hline Parameter Set \# & $\alpha$ & $\beta$ & $\gamma$ & $\begin{array}{l}\text { MAE } \\
\text { (ML) }\end{array}$ & $\begin{array}{l}\text { MAE } \\
\text { (FT) }\end{array}$ \\
\hline 1 & 0.1 & 0.1 & 0.8 & 0.739 & 1.381 \\
\hline$\vdots$ & $\vdots$ & $\vdots$ & $\vdots$ & $\vdots$ & $\vdots$ \\
\hline $\mathbf{2 9}$ & $\mathbf{0 . 5}$ & $\mathbf{0 . 3}$ & $\mathbf{0 . 2}$ & $\mathbf{0 . 7 3 2}$ & 1.378 \\
\hline$\vdots$ & $\vdots$ & $\vdots$ & $\vdots$ & $\vdots$ & $\vdots$ \\
\hline $\mathbf{3 5}$ & $\vdots$ & $\vdots$ & $\vdots$ & $\vdots$ & $\mathbf{1 . 3 7 3}$ \\
\hline 36 & $\mathbf{0 . 7}$ & $\mathbf{0 . 2}$ & $\mathbf{0 . 1}$ & 0.738 & $\mathbf{1 . 3}$ \\
\hline
\end{tabular}

this information. Adjusted cosine similarity [3] between two items is used for measuring the similarity over rating data. Vector similarity [2] between two items is used for measuring the similarity using demographic and feature vectors.

Step-2: Boosted similarity Boosted ${ }_{S i m}$ is defined by a function, $f_{\max }$ that combines $R_{I_{\text {Sim }}}, D_{I_{\text {Sim }}}, F_{I_{\text {Sim }}}$, $R_{D \text { Sim }}, D_{D \text { Sim }}$, and $F_{D \text { Sim }}$ over set of items in the training set. This function uses (5) for making prediction, and tries to maximize the utility. Formally, it can be specified as follows:

$f_{\text {max }}=\underset{f \in F}{\operatorname{argmax}} u\left(m_{i}, n_{j}\right): \forall m_{i} \in M^{T}, \forall n_{j} \in N^{T}$.

Equation (3) tells us to choose that function which maximizes the utility (i.e. reduces the $M A E$ ) of all users $\left(M^{T}\right)$ over set of items $\left(N^{T}\right)$ in the training set. Table I gives the different combination of functions checked over the training set with their respective lowest $M A E$ observed. It shows that a cascading hybrid setting in which rating and demographic correlation are applied over the candidate neighbour items found after applying the feature correlation gives the minimum error.

Let $C_{n_{t}}=\left\{c_{1}, c_{2}, \cdots, c_{k}\right\}$ be the set of $k$ candidate neighbours found after applying the feature similarity. We define the boosted similarity Boosted $_{\text {Sim }}$ by a linear combination of $F_{I S i m}$, $R_{D \text { Sim }}$, and $D_{D \text { Sim }}$ over the set of items in the training set as follows:

$$
\begin{array}{r}
\text { Boosted }_{\text {Sim }}\left(n_{t}, c_{i}\right)=\alpha \times F_{\text {ISim }} \\
+\beta \times R_{D \text { Sim }} \\
+\gamma \times D_{D \text { Sim }},
\end{array}
$$

where, $\alpha, \beta$, and $\gamma$ parameters represent the relative impact of three similarities. We assume $\alpha+\beta+\gamma$ $=1$ without the loss of generality.

Step-3: Predicting the rating $P_{m_{a}, n_{t}}$ for an active user $m_{a}$ on target item $n_{t}$ is made by using the following formula:

$$
P_{m_{a}, n_{t}}=\frac{\sum_{i=1}^{k}\left(\text { Boosted }_{\text {Sim }}\left(n_{t}, c_{i}\right) \times r_{m_{a}, c_{i}}\right)}{\sum_{i=1}^{k}\left(\mid \text { Boosted }_{\text {Sim }}\left(n_{t}, c_{i}\right) \mid\right)} .
$$

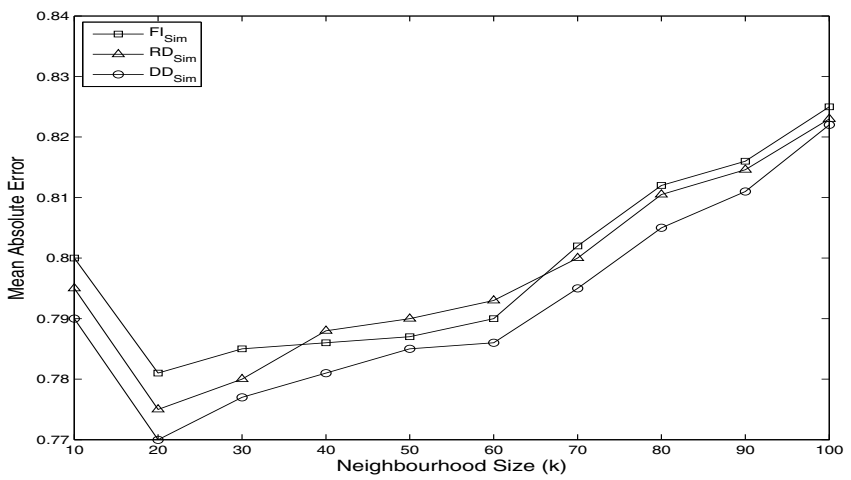

Figure 1. Determining the optimal value of neighbourhood size, $\mathrm{k}$ (MovieLens).

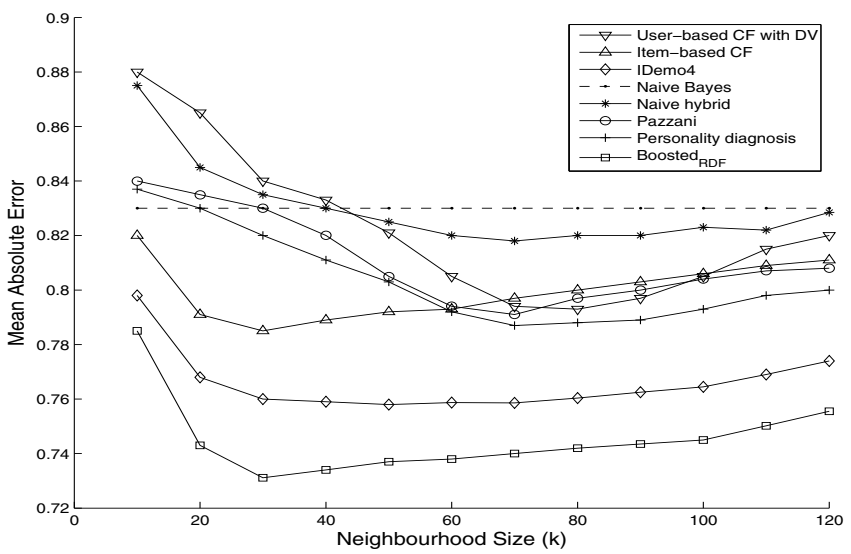

Figure 2. MAE of the proposed algorithm with others, against various neighbourhood sizes (MovieLens).

Equation (5) is the same as used by [3] except the rating similarity function has been replaced by Boosted $_{\text {Sim }}$.

\section{RESULT AND DiSCUSSION}

We randomly selected $20 \%$ ratings of each user as the test set and used the remaining $80 \%$ as training set. We further subdivided our training set into a test set and training set for measuring the parameters sensitivity. For learning the parameters, we conducted 5-fold cross validation on the $80 \%$ training set, by randomly selecting the different test and training set each time, and taking the average of results.

We compared our algorithm with seven different algorithms: user-based CF using Pearson correlation with default voting (DV) [2], item-based CF using adjustedcosine similarity [3], a hybrid recommendation algorithm, IDemo4, proposed in [7], a Naive Bayes classification approach using item features information, a naive hybrid approach for generating recommendation ${ }^{11}$, the personality diagnosis algorithm [11] for making probabilistic recommendations, and a hybrid recommendation algorithm used by Pazanni [6]. Furthermore, we tuned all algorithms for the best mentioning parameters.

\footnotetext{
${ }^{11}$ We take average of the prediction generated by a pure content-based and a pure user-based CF.
} 
Table III

A COMPARISON OF THE PROPOSED ALGORITHM WITH EXISTING IN TERMS OF COST (BASED ON [4], [11]), ACCURACY METRICS, AND COVERAGE

\begin{tabular}{|c|c|c|c|c|c|c|c|}
\hline Algorithm & On-line Cost & $\begin{array}{l}\text { Best } M A E \\
\text { (ML) }\end{array}$ & $\begin{array}{l}\text { Best } M A E \\
\text { (FT) }\end{array}$ & $\begin{array}{l}\text { ROC Sensitivity } \\
\text { (ML) }\end{array}$ & $\begin{array}{l}\text { ROC Sensitivity } \\
\text { (FT) }\end{array}$ & $\begin{array}{l}\text { Coverage } \\
\text { (ML) }\end{array}$ & $\begin{array}{l}\text { Coverage } \\
\text { (FT) }\end{array}$ \\
\hline User-based with DV & $N M^{2}$ & 0.791 & 1.441 & 0.401 & 0.643 & 99.424 & 93.611 \\
\hline Item-based & $N^{2}$ & 0.789 & 1.439 & 0.383 & 0.621 & 99.221 & 92.312 \\
\hline IDemo4 & $N^{2}$ & 0.768 & 1.415 & 0.430 & 0.644 & 99.572 & 94.441 \\
\hline Boosted $_{R D F}$ & $N^{2}$ & 0.725 & 1.362 & 0.562 & 0.756 & 100 & 99.195 \\
\hline Naive Bayes & $M(N P)$ & 0.831 & 1.470 & 0.622 & 0.835 & 100 & 99.997 \\
\hline Naive hybrid & $N M^{2}+M(N P)$ & 0.822 & 1.462 & 0.526 & 0.726 & 100 & 99.991 \\
\hline Pazzani & $N P M^{2}$ & 0.793 & 1.440 & 0.552 & 0.739 & 99.920 & 99.031 \\
\hline Personality diagnosis & $N M$ & 0.785 & 1.432 & 0.521 & 0.732 & 99.142 & 94.232 \\
\hline
\end{tabular}

\section{A. Locating the Optimal Value of Neighbourhood Size ( $k$ )}

We varied the number of neighbours for an active user, from 0 to 100 and computed the corresponding $M A E$ for $F_{I S i m}, R_{D S i m}$, and $D_{D S i m}$. The results are shown in Fig. 1.

Fig. 1 shows that for MovieLens dataset $M A E$ is minimum for $k=20^{12}$. We choose the neighbourhood size to be 20 for further experiments.

\section{B. Learning the Optimal Values of Parameters $(\alpha, \beta, \gamma)$}

The 36 parameter sets were generated by producing all possible combination of parameters values, ranging from 0.1 to 1.0 with differences of 0.1 . Table II presents a sample of the parameter sets learned. The parameters sets $\alpha=0.5, \beta=0.3, \gamma=0.2$, and $\alpha=0.7, \beta=0.2, \gamma=0.1$ gave the lowest $M A E$ in the case of MovieLens and FilmtTrust dataset respectively. It is worth noting that combined similarity depends heavily on feature similarity, i.e. $\alpha$. Furthermore, the values of parameters are found different for MovieLens and FilmTrust dataset, which is due to the fact that both dataset have different density, rating distribution, and rating scale.

\section{Comparison of the Proposed Algorithm with Others}

1) Performance Evaluation in Terms of MAE: Fig. 2 shows that our algorithm significantly outperforms other algorithms ${ }^{13}$. Similar results were observed in the case of FilmTrust dataset. We can conclude from the results that clusters of items found after applying $F_{\text {ISim }}, R_{D \text { Sim }}, D_{D \text { Sim }}$ have complementary role for prediction generation.

2) Comparison of MAE, ROC Sensitivity, Coverage, and On-line Cost of the Proposed Algorithm with Others: Table III shows the on-line cost (in the worst case) of each algorithm, with the corresponding lowest $M A E, R O C$ sensitivity, and coverage. Here, $P$ is the number of features against a training example (i.e. features against a movie). It is worth noting that for FilmTrust dataset, ROC sensitivity is higher, for all algorithms in general, as compared to the MovieLens dataset. We believe that it is due to the rating distribution ${ }^{14}$. Furthermore, the coverage of the algorithms

\footnotetext{
${ }^{12}$ The size found to be between $20-30$ for FilmTrust dataset.

${ }^{13}$ In Fig. 2, the $\mathrm{x}$-axis represents the number of neighbouring items in the case of item-based CF, IDemo4, and Boosted DemoFeature; and the number of neighbouring users otherwise.

${ }^{14}$ In FilmTrust, majority of the users have rated the popular set of movies and their rating tends to match the average rating of the movies.
}

Table IV

PERFormance Evaluation Under NeW ITEM COLD-START PROBLEM

\begin{tabular}{|l|l|l|l|l|l|l|}
\hline \multirow{2}{*}{ Algorithm } & \multicolumn{2}{|l|}{ MAE1 } & \multicolumn{2}{l|}{ MAE2 } & \multicolumn{2}{l|}{ MAE5 } \\
\cline { 2 - 7 } & (ML) & (FT) & (ML) & (FT) & (ML) & (FT) \\
\hline User-based CF with DV & 1.64 & 2.67 & 1.23 & 2.22 & 0.95 & 1.94 \\
Item-based CF & 1.34 & 2.51 & 1.18 & 2.13 & 0.91 & 1.58 \\
IDemo4 & 1.25 & 2.34 & 1.15 & 2.09 & 0.89 & 1.53 \\
Boosted $_{R D F}$ & 0.98 & 1.62 & 0.85 & 1.57 & 0.82 & 1.42 \\
\hline
\end{tabular}

is much lower in the case of FilmTrust dataset, which is due to the reason that it is very sparse $(99 \%)$. The table depicts that Boosted DemoFeature $_{\text {is scalable and practical }}$ as its on-line cost is less or equal to the cost of other algorithms.

3) Performance Evaluation Under New Item and New User Cold-Start Problems: When a new item is added to the system, then it is not possible to get rating for that item from significant number of users, and consequently the $\mathrm{CF}$ recommender system would not be able to recommend that item. This problem is called new item cold-start problem [1]. For testing our algorithm in this scenario, we selected 1000 random samples of user/item pairs from the test set. While making prediction for a target item, the number of users in the training set who have rated the target item were kept 1,2 , and 5 . The corresponding $M A E$; represented by $M A E 1, M A E 2, M A E 5$, is shown in the table IV. Table IV shows that proposed scheme works well in new item cold-start problem scenario, as it does not solely depend on the number of users who have rated the target item for finding the similarity.

For new user cold-start problem [2], where the profile of a user is incomplete, we use a linear regression model for finding an approximation of the active user's rating for an item. We use this rating instead of the active user's actual rating in (5) for prediction generation.

$$
r_{m_{a}, n_{i}}^{\prime}= \begin{cases}r_{m_{a}, n_{i}:} & \text { if active user rated more } \\ & \text { than } J \text { movies } \\ r_{r e g}: & \text { otherwise. }\end{cases}
$$

In 6 , the choice of $J$ came from the training set, which found to be 10 for MovieLens and 5 for FilmTrust dataset. Rating $r_{r e g}$ is found by a linear regression model: $R_{s}=$ $\theta_{1} R_{t}+\theta_{2}$, where $R_{s}$, and $R_{t}$ are the vector of similar item and vector of target item respectively ${ }^{15}$. Parameters $\theta_{1}$ and

\footnotetext{
${ }^{15}$ These vector are made up of all user who have rated that item in the training set. For more information, refer to [3].
} 


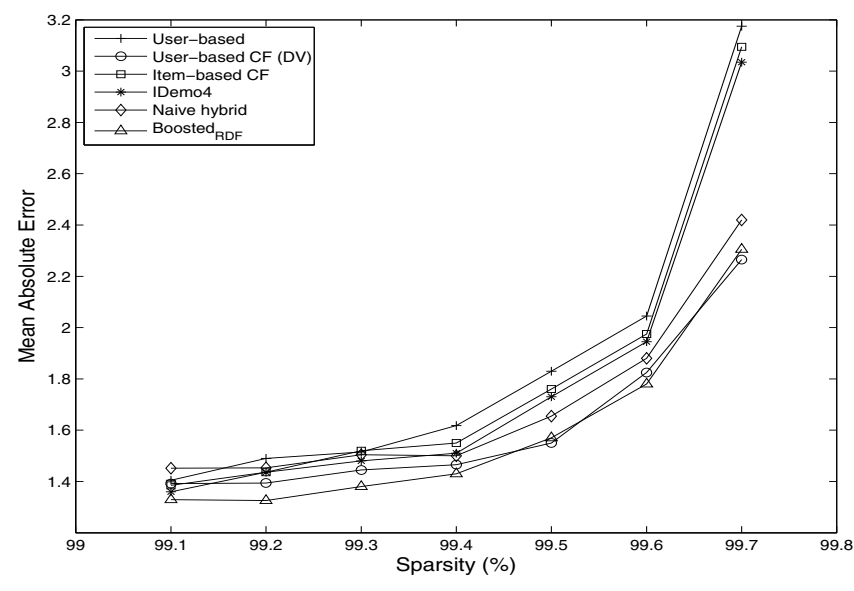

Figure 3. Performance of algorithms under different sparsity levels (FilmTrust).

$\theta_{2}$ can found by both rating vectors. This model was used in [3] for overcoming the misleading similarities between items in the item-based CF. We show this model makes sense to apply only when we have incomplete user profile. The performance of the test set in the case of Given 2 and Given $5^{16}$ [2] protocol is given in the table V.

Table $\mathrm{V}$ shows that our algorithm with regression gave more accurate results than others. User-based CF with DV gave good results, as it assumes some default votes for items a user has not voted on.

4) Performance Evaluation Under Different Sparsity Levels: To check the effect of sparsity, we increased the sparsity level of the training set by dropping some randomly selected entries. Whereas, we kept the test set same for each sparse training set. We checked the performance of the proposed algorithm with those of pure user-based CF, user-based CF with DV, item-based CF, IDemo4, and a naive hybrid recommendation algorithm. Fig. $3^{17}$ shows that performance does not degrades rapidly in the case of proposed algorithm. It is because; features of an item can still be used for finding similar items. Furthermore, synonym detection algorithm enrich item profiles while finding the similarity between the items.

\section{CONCLUSION}

In this paper, we have proposed a cascading hybrid recommendation approach by combining the feature correlation with rating and demographic information of an item. We showed empirically that our approach outperformed the state of the art algorithms.

\section{ACKNOWLEDGMENT}

The work reported in this paper has formed part of the Instant Knowledge Research Programme of Mobile VCE, (the Virtual Centre of Excellence in Mobile \& Personal Communications), www.mobilevce.com. The programme

\footnotetext{
${ }^{16}$ Given $\mathrm{N}$ means, active user has rated $\mathrm{N}$ items.

${ }^{17}$ Results were the same for MovieLens dataset. We show results for FilmTrust to analyse the performance of the algorithms under extreme sparsity (i.e. sparsity $>99 \%$ ).
}

Table V

PERFORMANCE EVALUATION UNDER NEW USER COLD-START PROBLEM

\begin{tabular}{|l|l|l|l|l|}
\hline \multirow{2}{*}{ Algorithm } & \multicolumn{2}{|l|}{ MAE: Given 2 } & \multicolumn{2}{c|}{ MAE: Given 5 } \\
\cline { 2 - 5 } & (ML) & (FT) & (ML) & (FT) \\
\hline User-based CF with DV & 1.06 & 2.03 & 0.86 & 1.48 \\
Item-based CF & 1.09 & 2.06 & 0.88 & 1.51 \\
IDemo4 & 1.07 & 2.05 & 0.85 & 1.48 \\
Boosted $_{R D F}$ & 1.06 & 2.01 & 0.83 & 1.47 \\
Boosted $_{R D F_{\text {Reg }}}$ & 1.01 & 1.93 & 0.79 & 1.41 \\
\hline
\end{tabular}

is co-funded by the UK Technology Strategy Boards Collaborative Research and Development programme. Detailed technical reports on this research are available to all Industrial Members of Mobile VCE. Thanks Martin for Providing the dataset.

\section{REFERENCES}

[1] A. T. Gediminas Adomavicius, "Toward the next generation of recommender systems: A survey of the state-of-the-art and possible extensions," IEEE Transactions on Knowledge and Data Engineering, vol. 17, 2005, pp. 734-749.

[2] J. S. Breese, D. Heckerman, and C. Kadie, "Empirical analysis of predictive algorithms for collaborative filtering," Morgan Kaufmann, 1998, pp. 43-52.

[3] B. Sarwar, G. Karypis, J. Konstan, and J. Reidl, "Itembased collaborative filtering recommendation algorithms," in Proceedings of the 10th international conference on World Wide Web. ACM New York, NY, USA, 2001, pp. 285-295.

[4] M. Vozalis and K. Margaritis, "Using SVD and demographic data for the enhancement of generalized collaborative filtering," Information Sciences, vol. 177, no. 15, 2007, pp. 3017-3037.

[5] R. Burke, "Hybrid recommender systems: Survey and experiments, User Modeling and User-Adapted Interaction," vol. 12, no. 4, 2002, pp. 331-370.

[6] M. J. Pazzani, "A framework for collaborative, content-based and demographic filtering," Artificial Intelligence Review, vol. 13, no. 5-6, 1999, pp. 393-408.

[7] M. Vozalis and K. Margaritis, "On the enhancement of collaborative filtering by demographic data," Web Intelligence and Agent Systems, vol. 4, no. 2, 2006, pp. 117-138.

[8] K. Lang, Newsweeder: "Learning to filter netnews," in Proceedings of the Twelfth International Conference on Machine Learning, 1995.

[9] P. Melville, R. J. Mooney, and R. Nagarajan, "Contentboosted collaborative filtering for improved recommendations," in Eighteenth National Conference on Artificial Intelligence, 2002, pp. 187-192.

[10] L. G. T. Jonathan L. Herlocker, Joseph A. Konstan and J. T. Riedl, "Evaluating collaborative filtering recommender systems," ACM Transactions on Information Systems (TOIS) archive, vol. 22, 2004, pp. 734-749.

[11] D. Pennock, E. Horvitz, S. Lawrence, and C. Giles, "Collaborative filtering by personality diagnosis: A hybrid memory and model-based approach," in Proceedings of the 16th Conference on Uncertainty in Artificial Intelligence, 2000, pp. 473-480. 